\title{
Evaluation of muscle quality and quantity for the assessment of sarcopenia using mid-thigh computed tomography: a cohort study
}

Hiroki Oba ${ }^{1,2^{*}}$ D, Yasumoto Matsui ${ }^{2}$, Hidenori Arai ${ }^{2}$, Tsuyoshi Watanabe ${ }^{2,3}$, Hiroki lida ${ }^{1,2}$, Takafumi Mizuno ${ }^{1,2}$, Satoshi Yamashita', Shinya Ishizuka', Yasuo Suzuki ${ }^{2,4}$, Hideki Hiraiwa' and Shiro Imagama'

\begin{abstract}
Background: For the diagnosis of Sarcopenia, European Working Group on Sarcopenia in Older People (EWGSOP) revised the algorisms in 2019, where they added computed tomography (CT) as an assessment tool not only for quantity but also for quality in research purpose. However, the evidence for clinical appreciation of CT has been lacking. Therefore, we investigated the correlation between CT and various motor function tests to assess the utility of $\mathrm{CT}$ as a potential diagnostic tool for sarcopenia.
\end{abstract}

Methods: In total, 214 patients who were examined at our center during the study period (2016-2017) were included in the study. Single-slice CT scan of the mid-thigh region was performed, from which cross-sectional area (CSA) and CT attenuation value (CTV) of quadriceps femoris were evaluated for each subject. Other assessments included skeletal muscle mass index by DXA and BIA, muscle strength and physical performance. Furthermore, subjects were classified into four groups as per the Asia Working Group of Sarcopenia (AWGS) 2019 criteria as those with: normal, poor muscle function/strength (poor function), sarcopenia and severe sarcopenia.

Results: Knee muscle strength correlated with CSA $(r=0.60)$ and the correlation was significantly greater than that with DXA and BIA. For physical performance, standing-up test correlated with CSA $(r=-0.20)$ and CTV $(r=-0.40)$ and walking speed with CTV $(r=0.43)$, which was significantly greater than that with DXA and BIA. The CSA was significantly lower in women with sarcopenia group and in both men and women with severe sarcopenia (all $p<$ 0.01). Furthermore, CTV was significantly lower in women with poor-function and in both men and women with severe sarcopenia group (all $p<0.01$ ).

Conclusions: CSA mostly correlated with muscle strength, whereas CTV mostly correlated with physical performance. CT with measurements of CSA and CTV enables the evaluation of muscle mass and quality simultaneously. CT is believed to be useful in inferring evaluation of motor function and assessment of sarcopenia.

Keywords: Sarcopenia, Mid-thigh computed tomography, Muscle quality, Computed tomography attenuation value, Motor function

\footnotetext{
* Correspondence: hiroki.o.0402@gmail.com

'Department of Orthopedics, Nagoya University Graduate School of Medicine, 65 Tsurumaicho, Showaku, Nagoya, Aichi 466-8560, Japan

${ }^{2}$ Center for Frailty and Locomotive Syndrome, National Center for Geriatrics and Gerontology, 7-430 Moriokacho, Obu, Aichi 474-8511, Japan

Full list of author information is available at the end of the article
}

(c) The Author(s). 2021 Open Access This article is licensed under a Creative Commons Attribution 4.0 International License, which permits use, sharing, adaptation, distribution and reproduction in any medium or format, as long as you give appropriate credit to the original author(s) and the source, provide a link to the Creative Commons licence, and indicate if changes were made. The images or other third party material in this article are included in the article's Creative Commons licence, unless indicated otherwise in a credit line to the material. If material is not included in the article's Creative Commons licence and your intended use is not permitted by statutory regulation or exceeds the permitted use, you will need to obtain permission directly from the copyright holder. To view a copy of this licence, visit http://creativecommons.org/licenses/by/4.0/ The Creative Commons Public Domain Dedication waiver (http://creativecommons.org/publicdomain/zero/1.0/) applies to the data made available in this article, unless otherwise stated in a credit line to the data. 


\section{Background}

As the adult population ages, the extension of healthy life expectancy has become a major issue. Sarcopenia was initially defined as a reduction in the skeletal muscle mass with aging. The term "sarcopenia" was first introduced by Rosemberg et al. [1] in 1989, from the Greek terms "sarx" (muscle) and "penia" (loss). In 1998, Baumgartner et al. [2] defined sarcopenia as a mean skeletal muscle mass index (SMI) of -2SD or less determined using dual energy X-ray absorption (DXA) in young individuals, and since then, sarcopenia has been determined using SMI. However, it has been reported that compared with muscle mass, muscle strength is more associated with falling and limited mobility [3, 4], and therefore, in determining sarcopenia, it is thought that muscle strength and physical performance should be included. The 2010 consensus report from the European Working Group on Sarcopenia in older people (EWGSOP) [5] proposed a diagnostic algorithm for determining sarcopenia according to SMI, grip strength, and walking speed. In 2011, a condition "sarcopenia with limited mobility" defined as a person with muscle loss whose walking ability is reduced was described as a condition that requires treatment [6].

In 2014, the Asia Working Group of Sarcopenia (AWGS) presented diagnostic criteria for sarcopenia based on data obtained from various Asian countries [7]. Furthermore, In the EWGSOP2 revised in 2018, attention was focused on loss of muscle strength as the primary parameter of sarcopenia, and for a definite diagnosis of sarcopenia, it was reported that the assessment of muscle quantity and quality is important [8]. AWGS 2019 updated the consensus of sarcopenia diagnosis in Asia: the previous definition of sarcopenia was retained; however, the diagnostic algorithm, protocols, and some criteria have been revised. Specifically, AWGS 2019 introduces "possible sarcopenia," defined by low muscle strength with or without reduced physical performance [9].

Muscle mass is conventionally measured using DXA and bioelectrical impedance analysis (BIA). DXA can measure appendicular skeletal muscle mass; however, there are problems with this method, i.e., the values differ depending on the machine used [10], and in Asian women, this measurement does not change with age [11]. BIA is affected by the amount of body fluids; therefore, precise evaluations cannot be achieved [12]. Therefore, the EWGSOP2 described computed tomography (CT) and magnetic resonance imaging (MRI) as methods to verify the muscle quantity and quality; however, there are only few reports of their use in clinical practice [13].

Motor function is evaluated using measurements of walking speed and grip strength; however, the reproducibility is poor depending on the physical and mental condition of the participant and the measurement methods and tool used [14]. CT and MRI are considered the gold standards of minimally invasive tests to measure muscle mass [15]; however, their use in the evaluation of muscle quality remains controversial. Goodpaster et al. [16] noted a good correlation between CT attenuation value (CTV) and intramuscular fat infiltration, whereas Ikemoto-Uezumi et al. [17] reported reduced muscle fibers and fatty tissue infiltration in the muscular tissue of the vastus medialis in patients with osteoarthritis. Therefore, it is inferred that CT can evaluate muscle quantity and that CTV can evaluate fat infiltration which might be associated with decreased motor function.

However, there are few reports of the use of CTV in CT scans of the mid-thigh and their use in evaluating motor function. The purpose of this study was to evaluate to what extent CT, DXA and BIA derived muscle parameters correlate with muscle strength and function in order to investigate the utility of CT as a potential assessment method for muscle quality as well as for the assessment of sarcopenia.

\section{Methods}

Of 300 patients who were examined at the outpatient services of the National Center for Geriatrics and Gerontology, Center for Frailty and Locomotive Syndrome between 2016 and 2017, 214 (78 men and 136 women; average age, 78.3 and 78.4 years respectively) were included in the study after excluding those with gait impairment caused by severe osteoarthritis of the knee and hip, progressive motor disease such as Parkinson disease, dementia and those with missing data. To assess the risk of falls, we investigated fall scores, as proposed by Toba et al. [18] To assess motor function, we measured walking speed (Walkway MW-1000, ANIMA, Tokyo Japan); grip strength using a new grip dynamometer developed at our institution with ZP-500 N (Imada Co., Ltd., Toyohashi, Aichi, Japan) [19]; knee extension strength (ZP500 N, Imada Co., Ltd., Toyohashi, Aichi, Japan) that is the measure of the isometric muscle extension strength in the sitting position with knee at $90^{\circ}$ of flexion, developed at our institution [20]; single-leg standing; timed up and go (TUG); short physical performance battery (SPPB) using stand-up test and two-step test. For single leg standing, the participants were instructed to stand on a single leg with their eyes open as long as possible and the time was recorded. For TUG, we measured the time required to stand up from the chair and go back 3 $\mathrm{m}$ and to sit on the chair again. The stand-up and twostep tests were performed to assess the degree of locomotive syndrome. For the stand-up test, we measured whether the participants could stand up on both the legs from the sitting position on a platform of $10,20,30$, and 
$40 \mathrm{~cm}$ [21]. For the two-step test, the participants stood at the starting line and took two strides as long as possible; then, the two legs were aligned and the distance of the two steps was measured. For SPPB, the test evaluated the balance, gait, strength, and endurance by examining an individual's ability to stand on their feet together in side-by-side semi-tandem and tandem positions, time to walk $8 \mathrm{ft}$., and time to rise from a chair and return to the seated position five times [22]. Furthermore, the muscle mass of the four limbs from the whole-body scan was measured by DXA (Lunar iDXA, GE Healthcare, Chicago, Illinois, USA) and BIA (Inbody720, BIOSPACE, Iowa, USA). Appendicular skeletal muscle mass (ASM) (kg) by DXA and BIA / height $\mathrm{t}^{2}\left(\mathrm{~m}^{2}\right)$ was calculated as a skeletal muscle mass index (SMI) [2].

For CT (SOMATOM Sensation 64; Siemens, Munich, Germany), the subject was placed in the supine position, and a single slice of the right mid-thigh was taken. We defined the mid-thigh as the midpoint of the inguinal ligament and the superior pole of the patella manually. The scanning conditions were as follows: $120 \mathrm{kV}, 120$ $\mathrm{mA}$, rotation time of $1 \mathrm{~s}$, and field of view of $233 \mathrm{~mm}$. From this single slice, the cross-sectional surface area (CSA) and CTV of the quadriceps femoris muscle were measured using SliceOmatic ver.5.0 (Tomovision, Canada) by a single examiner (Fig. 1). CTV was defined as -1000 Hounsfield Unit (HU) for air and $0 \mathrm{HU}$ for water and indicated the degree of X-ray absorption of a substance. The average CTV is $40-100 \mathrm{HU}$ for a muscle and approximately -50 to $-100 \mathrm{HU}$ for fat. For CSA and CTV, the imaging data of 21 subjects were measured by three examiners, and the associated interclass correlation was determined. The intraclass correlation of CSA and CTV of the examiners was $>0.998$, and the interclass correlation among the three examiners was 0.999 .
The subjects were classified in accordance with the AWGS 2019 diagnostic criteria [7] into four groups. Among the subjects without reduced walking speed and grip strength, the group with normal muscle mass was defined as the "normal" group and the group with reduced walking speed and/or grip strength and normal muscle mass was defined as the "poor muscle function/ strength (poor function)" group. The group with low ASM and low muscle strength or low physical performance was termed the "sarcopenia" group, and the group with low ASM, low muscle strength, and low physical performance was called the "severe-sarcopenia" group.

According to the AWGS 2019 criteria, the cutoff values [7] were as follows: walking speed, $1.0 \mathrm{~m} / \mathrm{s}$; grip strength, $28 \mathrm{~kg}$ for men and $18 \mathrm{~kg}$ for women; SMI with DXA $7.0 \mathrm{~kg} / \mathrm{m}^{2}$ for men and $5.4 \mathrm{~kg} / \mathrm{m}^{2}$ for women; and SMI with BIA $7.0 \mathrm{~kg} / \mathrm{m}^{2}$ for men and $5.7 \mathrm{~kg} / \mathrm{m}^{2}$ for women. For the four groups, we evaluated the association between the muscle function and CT, DXA and BIA.

Statistical analysis were performed via SPSS (IBM SPSS Statistics ver.24, Tokyo, Japan) using ANOVA for demographic data for men and women, CSA and CTV of the quadriceps femoris muscle between each four groups. Tukey's test was applied for post-hoc multiple testing. To determine the association between muscle strength and function parameters on one hand, and CSA, CTV, DXA and BIA on the other hand, partial correlations were calculated to correct for the effect of gender. Pearson's correlation coefficient was applied for muscle strength and Spearman's correlation coefficient was applied for physical performance test. The difference of correlation coefficient among the subjects was calculated using Fisher's $\mathrm{Z}$ transformation [23], and then Bonferroni correction was performed. $P$ values of $<0.05$ were considered statistically significant. Power analysis was performed via G*Power (Heinrich Heine university, a

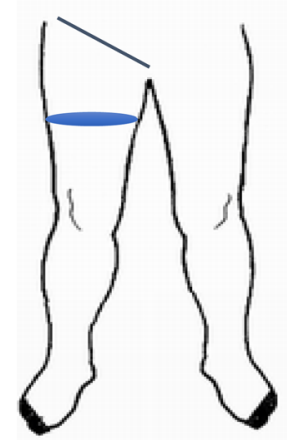

b

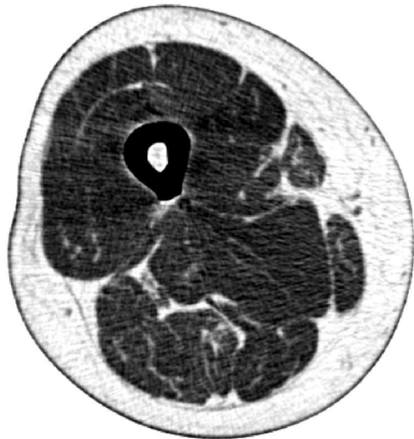

C

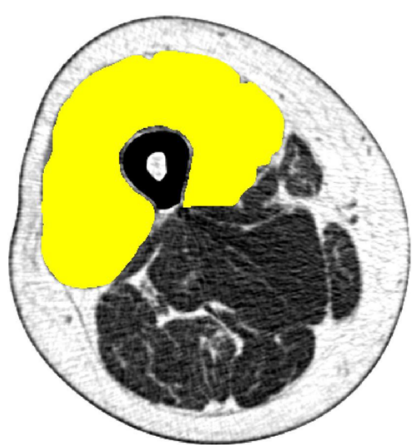

Fig. 1 Procedure for $C T$ measurement. a. We defined the mid-thigh as the midpoint of the inguinal ligament and the superior pole of the patella manually. b. Obtained CT image of mid-thigh. c. Trace quadriceps femoris using SliceOmatic manually. CT: computed tomography 
version 3.1.9.7, Dusseldorf, Germany). The parameters were as follows: $\alpha=0.05,1-\beta=0.8$, assumed correlation coefficients between CSA and grip strength was 0.2 , CTV and grip strength was 0.4 and CTV and CSA was 0.3 [24]. Because the calculated appropriate sample size was 236 , the sample size was almost sufficient for the study.

\section{Results}

Overall, 99 subjects were included in the normal group, 55 in the poor-function group, 26 in the sarcopenia group, and 34 in the severe sarcopenia group. Compared with the normal group, age and the fall risk index were significantly higher in the poor-function, severe sarcopenia groups in women (Table 1).

Knee extension strength and grip strength were correlated with CSA, BIA, DXA and CTV. CSA had significantly stronger correlation with knee extension strength than BIA and DXA, whereas CTV did not have significantly stronger correlation with others (Table 2).

In contrast, in terms of the physical performance measurements, the correlation coefficients for CSA and CTV showed that these were correlated with single leg standing, walking speed, standing up test, TUG, SPPB, and two-step test. CTV showed a significantly stronger correlations with walking speed compared with BIA, and a significantly stronger correlation with the standing up test compared with both BIA and DXA (Table 2).

About CSA and CTV, three abnormal groups (poorfunction, sarcopenia and severe sarcopenia groups) were compared with normal group. CSAs of the quadriceps femoris muscle were significantly smaller in the severe sarcopenia groups $(P<0.001)$ for men and significantly smaller in the sarcopenia $(\mathrm{P}<0.001)$, and severe sarcopenia groups $(\mathrm{P}<0.001)$ for women (Fig. 2). Whereas, CTVs were significantly lower in the severe sarcopenia group $(P=0.001)$ for men and significantly lower in the poor-function $(P=0.008)$ and severe sarcopenia groups $(\mathrm{P}<0.001)$ for women (Fig. 2).

\section{Discussion}

In this study, we determined the correlation between CT and various motor function, using different assessment methods for muscle quantity / quality, including CT, BIA, and DXA. The result indicated that CSA showed the most correlation with muscle strength, whereas CTV showed the most correlation with physical performance.

The concept of sarcopenia has considerably changed over time, and recently, the EWGSOP2 has stated that the importance of measuring muscle quality is expected to grow as a defining feature of sarcopenia; however, there is no universal consensus on the assessment method for routine clinical practice [8]. Patients with dynapenia and sarcopenia which are considered as conditions of low muscle quality are at a high risk of falling [3]. In accordance with the present study, patients with poor function and sarcopenia had a significantly higher risk of falling, which was believed to be associated with reduced motor function. There is a physical state in which muscle mass is maintained but with reduced functioning and muscle quality. McGregor et al. [25] referred that not only changes in muscle mass but also other factors underpinning muscle quality including composition, metabolism, aerobic capacity, insulin resistance, fat infiltration, fibrosis, and neural activation may play a role in the decline in muscle function and impaired mobility associated with aging.

To date, muscle quality is determined by evaluating intramuscular fat infiltration using highly sensitive measurement devices such as CT and MRI [26]. Moreover, in the recent years, muscle quality has been evaluated using ultrasound; however, there is no consensus for any of

Table 1 Demographic data by group

\begin{tabular}{|c|c|c|c|c|c|c|c|c|c|c|c|c|c|}
\hline \multirow[t]{2}{*}{ Group } & \multirow[b]{2}{*}{$\mathbf{n}$} & & & \multicolumn{2}{|c|}{ Age (year) } & \multicolumn{2}{|c|}{ Height $(\mathrm{cm})$} & \multicolumn{2}{|c|}{ Weight (kg) } & \multicolumn{2}{|c|}{$\mathrm{BMI}\left(\mathrm{kg} / \mathrm{cm}^{2}\right)$} & \multicolumn{2}{|c|}{ Fall risk score } \\
\hline & & & & average & SD & average & SD & average & SD & average & SD & average & SD \\
\hline \multirow[t]{2}{*}{ Normal } & 99 & M & 36 & 77.0 & 5.0 & 163.6 & 4.7 & 60.8 & 7.0 & 22.7 & 2.5 & 7.3 & 3.1 \\
\hline & & $\mathrm{F}$ & 63 & 75.6 & 5.1 & 150.7 & 5.4 & 52.3 & 9.7 & 23.0 & 3.9 & 7.9 & 3.2 \\
\hline \multirow[t]{2}{*}{ Poor-function } & 55 & M & 13 & 77.7 & 5.1 & 159.3 & 8.5 & 67.9 & 12.1 & 26.6 & 3.1 & 9.2 & 3.2 \\
\hline & & $\mathrm{F}$ & 42 & $78.9^{*}$ & 5.3 & 146.7 & 7.2 & 53.9 & 10.1 & $25.0^{*}$ & 3.8 & $11.0^{*}$ & 3.0 \\
\hline \multirow[t]{2}{*}{ Sarcopenia } & 26 & M & 14 & 79.9 & 5.8 & 159.9 & 4.9 & 55.6 & 7.6 & 21.7 & 2.2 & 8.8 & 2.9 \\
\hline & & $\mathrm{F}$ & 12 & $81.8^{*}$ & 3.5 & 148.7 & 4.9 & $41.5^{*}$ & 5.6 & $18.8^{*}$ & 2.5 & $10.4^{*}$ & 2.2 \\
\hline \multirow[t]{2}{*}{ Severe Sarcopenia } & 34 & M & 15 & 81.6 & 7.9 & 161.6 & 7.7 & 54.1 & 9.7 & 20.7 & 3.5 & $10.1^{*}$ & 3.4 \\
\hline & & $F$ & 19 & $83.6^{*}$ & 4.7 & 143.6 & 5.7 & $39.6^{*}$ & 5.6 & $19.1^{*}$ & 1.6 & $10.7^{*}$ & 2.8 \\
\hline \multirow[t]{2}{*}{ Total } & 214 & M & 78 & 78.5 & 6.0 & 161.8 & 6.3 & 59.7 & 9.6 & 22.8 & 3.3 & 8.4 & 3.3 \\
\hline & & $\mathrm{F}$ & 136 & 78.3 & 5.7 & 148.3 & 6.5 & 50.1 & 10.5 & 22.7 & 4.1 & $9.5^{*}$ & 3.3 \\
\hline
\end{tabular}

${ }^{*} P<0.05$ compared with normal group, $M$ men, $W$ women, $B M I$ body mass index, $S D$ standard deviation

Age was significantly higher in the poor-function, sarcopenia and severe-sarcopenia groups than in the normal group 
Table 2 Correlation between each method and motor function (muscle strength and physical performance) test

\begin{tabular}{|c|c|c|c|c|c|c|c|c|c|c|c|c|}
\hline & \multicolumn{3}{|l|}{ CSA } & \multicolumn{3}{|l|}{ BIA } & \multicolumn{3}{|l|}{ DXA } & \multicolumn{3}{|l|}{ CTV } \\
\hline & \multirow[t]{2}{*}{$r$} & \multicolumn{2}{|l|}{$95 \% \mathrm{Cl}$} & \multirow[t]{2}{*}{$r$} & \multicolumn{2}{|l|}{$95 \% \mathrm{Cl}$} & \multirow[t]{2}{*}{$r$} & \multicolumn{2}{|l|}{$95 \% \mathrm{Cl}$} & \multirow[t]{2}{*}{$r$} & \multicolumn{2}{|l|}{$95 \% \mathrm{Cl}$} \\
\hline & & Lower & Upper & & Lower & Upper & & Lower & Upper & & Lower & Upper \\
\hline \multicolumn{13}{|l|}{ Muscle strength } \\
\hline Knee extension strength & 0.60 & 0.56 & 0.83 & $0.391^{*}$ & 0.28 & 0.55 & $0.39^{*}$ & 0.27 & 0.54 & 0.26 & 0.13 & 0.40 \\
\hline Grip strength & 0.44 & 0.34 & 0.61 & 0.29 & 0.16 & 0.43 & 0.39 & 0.28 & 0.55 & 0.26 & 0.13 & 0.40 \\
\hline \multicolumn{13}{|l|}{ Physical performance } \\
\hline Single-leg standing & 0.24 & 0.11 & 0.36 & 0.18 & 0.05 & 0.31 & 0.20 & 0.07 & 0.33 & 0.24 & 0.11 & 0.37 \\
\hline Walking speed & 0.34 & 0.21 & 0.45 & $0.22+$ & 0.09 & 0.35 & 0.29 & 0.17 & 0.41 & 0.43 & 0.31 & 0.53 \\
\hline Standing up test & -0.20 & -0.33 & -0.07 & $0.01^{*}+$ & -0.12 & 0.15 & $0.05^{*} \dagger$ & -0.08 & 0.19 & -0.40 & -0.51 & -0.28 \\
\hline TUG & -0.38 & -049 & -0.26 & -0.27 & -0.40 & -0.14 & -0.30 & -0.42 & -0.18 & -0.34 & -0.45 & -0.21 \\
\hline SPPB (total) & 0.36 & 0.24 & 0.48 & 0.23 & 0.10 & 0.36 & 0.26 & 0.13 & 0.38 & 0.35 & 0.22 & 0.46 \\
\hline Two-step test & 0.33 & 0.21 & 0.45 & 0.24 & 0.11 & 0.37 & 0.31 & 0.19 & 0.43 & 0.40 & 0.28 & 0.51 \\
\hline
\end{tabular}

CSA cross-sectional area of quadriceps femoris measured by computed tomography (CT) scan, CTV CT attenuation value of quadriceps femoris measured by CT scan, $r$ Pearson's partial correlation coefficient for muscle strengths and Spearman's partial correlation coefficient for muscle performances, BIA Skeletal muscle Mass Index (SMI) determined by bioelectrical impedance analysis, DXA SMI determined by dual energy X-ray absorption

${ }^{*} P<0.05$ compared with CSA, $+P<0.05$ compared with CTV. ${ }^{*}$ ort means that CSA or CTV has significantly stronger correlation than the value. CSA showed the most association with muscle strength, whereas CTV showed the most association with physical performance

these assessments. There are few reports wherein CT of the mid-thigh has been used to evaluate the relationship of CSA and CTV using motor function. The present study was performed to evaluate whether CTV correlates with motor function, and whether it can be used to evaluate muscle quality.

In previous studies, parameters of simple muscle strength, such as grip [27] and quadriceps femoris muscle strength [28], have been correlated with CSA; in a similar manner, our study showed that the strongest correlation was that of CSA with grip and knee extension strength. Furthermore, in the evaluation of physical performance test that combined physical movement (single-leg standing, walking speed, stand-up test, TUG, SPPB, and two-step test), the highest correlation was observed with CTV, and walking speed and stand-up test showed a significantly stronger correlation with CTV than other inspection devices, suggesting that physical performance is associated with muscle quality as determined by CTV.

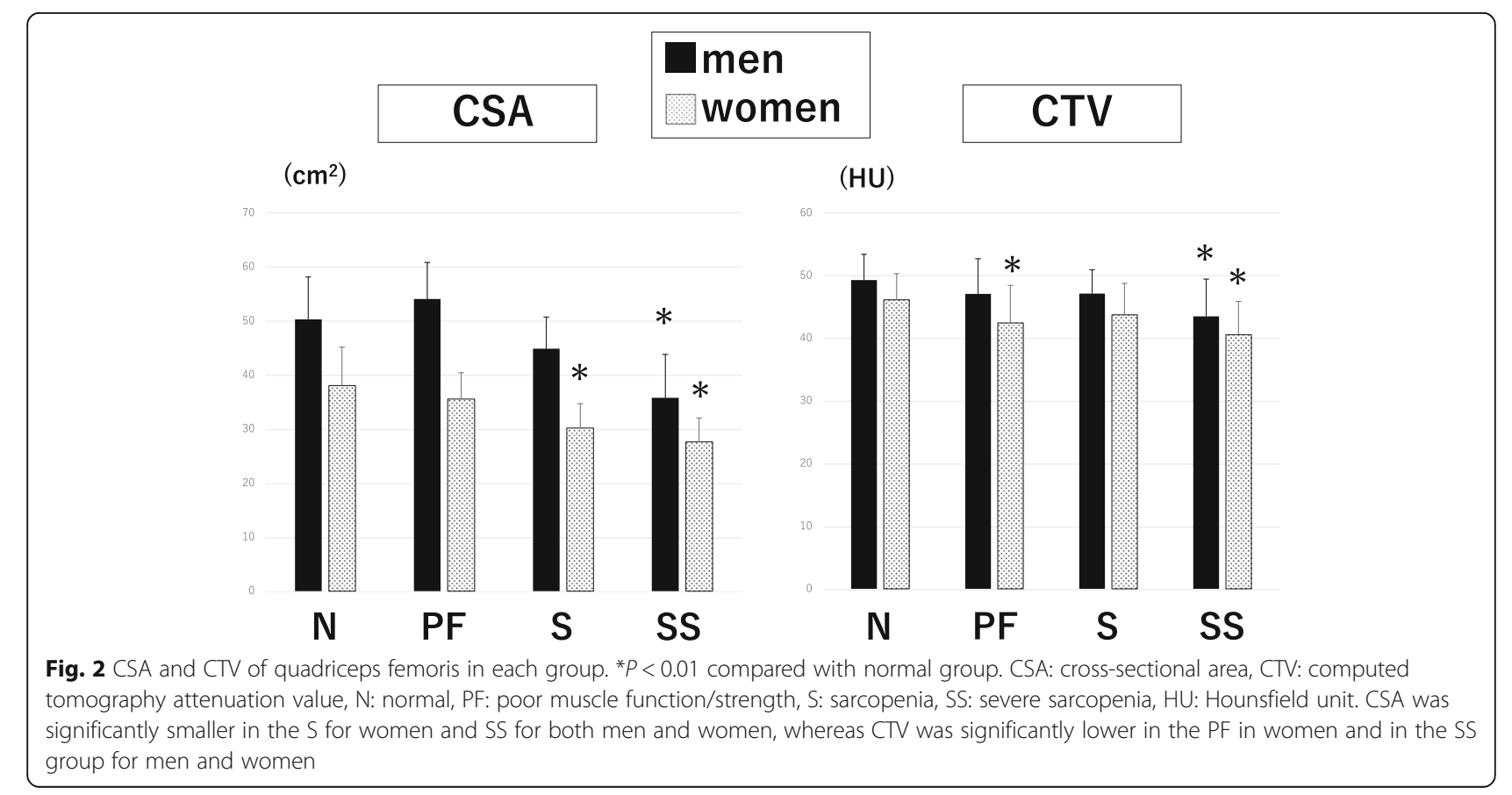


Lang et al. [29] reported that a low CTV of the thigh indicated a higher risk of fall and hip fracture; the results of the present study are consistent with these results. In this study, we classified patients into four groups as per muscle mass and motor function, based on the AWGS 2019 criteria. Between the women in the normal and sarcopenia groups, there was no decrease in the CTV; however, there was a significant decline in the CTV of those in the poor-function and severe sarcopenia groups. We consider that the decrease in CTV may be attributable to muscle atrophy and increased fat composition in the muscle. Ikemoto-Uezumi et al. [17] compared tissue in the vastus medialis muscle of patients with osteoarthritis and reported that intramuscular adipose tissue (IMAT) and an increased proportion of interstitial tissue were observed. Thus, a decline in CTV with an increased proportion of IMAT was associated with intramuscular fat infiltration [30]. Similarly, in sarcopenia, increased IMAT is observed [16], and these changes in muscle tissue could explain the decrease in CTV. Reportedly, increased IMAT measured by MRI is a prognostic factor of gait ability [31], and IMAT accumulates markedly after reduced activity in healthy young adults [32]. We consider that reduced activity or motor function may have caused an increase in IMAT and that CTV could evaluate this change in muscle composition.

As a minimally invasive tool, CT is considered as a gold standard to measure muscle quantity; however, it is not used in general practice owing to the high costs, poor portability, and requirement of an experienced operator [15]. In patients with cancer, reduced muscle mass is an independent predictor of immobility and mortality [33]. Kasai et al. [34] reported that among the muscles in the mid-thigh CT, CSA decreased with age mainly in quadriceps femoris. Schweitzer et al. [35] reported that on MRI, the best estimates for skeletal muscle were shown in the thigh. Lee SJ reported that CT of the femur was a useful method to evaluate muscle mass for the entire body [36]. Compared with other test equipment, DXA showed no change with age among Asian women [11]. Recently, we reported significant associations of thigh CT CSA with muscle strength [37]. For BIA, changes associated with the physical condition such as fluid balance and body temperature fluctuated during the day, resulting in reduced accuracy of BIA [38]. CT could be a better form of assessment because of its accuracy, reproducibility, and objectivity [39]. CT can simultaneously measure the muscle CSA and CTV, indicating muscle mass and composition; thus, CT is useful for assessing the severity of sarcopenia. Regarding the amount of radiation exposure, as only a single slice is scanned, we consider that there is no problem in terms of the radiation levels [10]. In addition, single slice leg CT scanning takes only about $5 \mathrm{~min}$. We think that the present results represent the initial steps toward the accumulation of evidence for the evaluation of muscle quality and will form the basis for future studies to examine the pathophysiology of severe sarcopenia accompanied by not only muscle mass loss but also physical functional decline. It is also useful for detailed evaluation in patients who have difficulty to walk, or to confirm the muscle condition after screening with a simple physical test.

There were limitations to the present study. First, this was a cross-sectional study and changes caused by natural history or therapeutic intervention could not be evaluated. Further study is required to determine whether or not the changes occurred as a result of exercise therapy, nutritional counseling, and pharmacotherapy. Second, this study did not include large-scale data of CSA and CTV from each age group of the general population without sarcopenia, and therefore, the healthy control information was weak. Thus, analysis using a large-scale epidemiological study is required. In the future, we plan to perform an analysis on a larger sample. As noted in the EWGSOP2, our results showed that CT may be useful in objectively evaluating sarcopenia more accurately and understand the pathology in greater detail [8].

\section{Conclusions}

This study was the first to report a relationship between mid-thigh CT and motor function in clinical setting. CT allows CSA and CTV to be measured simultaneously; CSA showed the most association with muscle strength, whereas CTV showed the most association with physical performance. These results could be useful for the assessing the presence and severity of sarcopenia.

\section{Abbreviations \\ AWGS: Asia Working Group of Sarcopenia; BIA: Bioelectrical impedance analysis; CSA: Cross-sectional area; CT: Computed tomography; CTV: CT Attenuation value; DXA: Dual energy X-ray absorption; EWGSOP: European Working Group on Sarcopenia in Older People; MRI: Magnetic resonance imaging; SMI: Skeletal muscle mass index; SPPB: Short physical performance battery; TUG: Timed Up and Go; HU: Hounsfield unit}

\section{Acknowledgments}

We would like to express our gratitude to everyone who cooperated in the study at National Center for Geriatrics and Gerontology.

\section{Authors' contributions \\ HO drafted the manuscript. YM suggested this study concept and design. HA supervised study concept and helped in the interpretation of this study. YS analyzed mid-thigh CT data and calculated CSA and CTV. HO and SY ana- lyzed the participants data. TW, HI, TM, SI1, HH and SI2 advised study design and results. All authors critically revised the report, commented on drafts of the manuscript, and approved the final manuscript.}

\section{Funding}

This work was supported by a grant from Expenses for Research and Development of Geriatrics and Gerontology (26-12, 29-12) and Furuno Electronics; the funding agencies played no role in the study. 


\section{Availability of data and materials}

The datasets generated during and/or analyzed during the current study are available from the corresponding author on reasonable request.

\section{Declarations}

\section{Ethics approval and consent to participate}

The study was conducted with the approval of the Ethical Review Board of the National Center for Geriatrics and Gerontology, Japan and all participants provided fully informed consent with written documents prior to participation.

\section{Consent for publication}

Not applicable.

\section{Competing interests}

The authors declare that they have no competing interests.

\section{Author details}

'Department of Orthopedics, Nagoya University Graduate School of Medicine, 65 Tsurumaicho, Showaku, Nagoya, Aichi 466-8560, Japan. ${ }^{2}$ Center for Frailty and Locomotive Syndrome, National Center for Geriatrics and Gerontology, 7-430 Moriokacho, Obu, Aichi 474-8511, Japan. ${ }^{3}$ Department of Orthopedics, National Center for Geriatrics and Gerontology, 7-430 Moriokacho, Obu, Aichi 474-8511, Japan. ${ }^{4}$ Department of Human Care Engineering, Faculty of Health Sciences, Nihon Fukushi University, Okuda, Mihamacho, Chita, Aichi 470-3295, Japan.

\section{Received: 22 August 2020 Accepted: 2 April 2021}

\section{Published online: 13 April 2021}

\section{References}

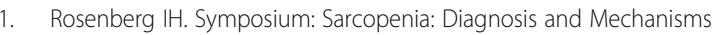
Sarcopenia: Origins and Clinical Relevance. 1997;127(5):990S-1S. https://aca demic.oup.com/jn/article-abstract/127/5/990S/4724144.

2. Baumgartner RN, Koehler KM, Gallagher D, Romero L, Heymsfield SB, Ross $\mathrm{RR}$, et al. Epidemiology of sarcopenia among the elderly in New Mexico. Am J Epidemiol. 1998;147(8):755-63. https://doi.org/10.1093/oxfordjournals.a je.a009520.

3. Schaap LA, Van Schoor NM, Lips P, Visser M. Associations of sarcopenia definitions, and their components, with the incidence of recurrent falling and fractures: the longitudinal aging study Amsterdam. J Gerontol A Biol Sci Med Sci. 2018;73(9):1199-204. https://doi.org/10.1093/gerona/gl×245.

4. Alley DE, Shardell MD, Peters KW, McLean RR, Dam TT, Kenny AM, et al. Grip strength cutpoints for the identification of clinically relevant weakness. Gerontol A Biol Sci Med Sci. 2014;69(5):559-66. https://doi.org/10.1093/ gerona/glu011.

5. Cruz-Jentoft AJ, Baeyens JP, Bauer JM, Boirie Y, Cederholm T, Landi F, et al. Sarcopenia: European consensus on definition and diagnosis: report of the European working group on sarcopenia in older people. Age Ageing. 2010; 39(4):412-23. https://doi.org/10.1093/ageing/afa034.

6. Morley JE, Abbatecola AM, Argiles JM, Baracos V, Bauer J, Bhasin S, et al. Sarcopenia with limited mobility: an international consensus. J Am Med Dir Assoc. 2011;12(6):403-9. https://doi.org/10.1016/j.jamda.2011.04.014.

7. Chen LK, Liu L, Woo J, Assantachai P, Auyeung T, Bahyah KS, et al. Sarcopenia in Asia: consensus report of the Asian working group for sarcopenia. J Am Med Dir Assoc. 2014;15(2):95-101. https://doi.org/10.1016/ j.jamda.2013.11.025.

8. Cruz-Jentoft AJ, Bahat G, Bauer J, Boirie Y, Bruyere O, Cederholm T, et al. Sarcopenia: revised European consensus on definition and diagnosis. Age Ageing. 2019;48(1):16-31. https://doi.org/10.1093/ageing/afy169.

9. Chen LK, Liu LK, Woo J, Assantachai P, Auyeung TW, Bahyah SK, et al. Asian working group for sarcopenia. J Am Med Dir Assoc. 2014;15(2):95-101. https://doi.org/10.1016/j.jamda.2013.11.025.

10. Buckinx F, Landi F, Cesari M, Fielding RA, Visser M, Engelke $K$, et al. Pitfalls in the measurement of muscle mass: a need for a reference standard. J Cachexia Sarcopenia Muscle. 2018;9(2):269-78. https://doi.org/10.1002/ jcsm. 12268

11. Shimokata H, Ando F, Yuki A, Otsuka R. Age-related changes in skeletal muscle mass among community-dwelling Japanese: a 12-year longitudinal study. Geriatr Gerontol Int. 2014;14(Suppl 1):85-92. https://doi.org/10.1111/ ggi.12219.

12. Gonzalez MC, Heymsfield SB. Bioelectrical impedance analysis for diagnosing sarcopenia and cachexia: what are we really estimating? J Cachexia Sarcopenia Muscle. 2017;8(2):187-9. https://doi.org/10.1002/ jcsm.12159.

13. Mourtzakis M, Prado CM, Lieffers JR, Reiman T, McCargar LJ, Baracos VE. A practical and precise approach to quantification of body composition in cancer patients using computed tomography images acquired during routine care. Appl Physiol Nutr Metab. 2008;33(5):997-1006. https://doi.org/1 $0.1139 / \mathrm{H} 08-075$

14. Beaudart C, Rolland Y, Cruz-Jentoft AJ, Bauer JM, Sieber C, Cooper C, et al. Assessment of muscle function and physical performance in daily clinical practice. Calcif Tissue Int. 2019;105(1):1-14. https://doi.org/10.1007/s00223019-00545-w.

15. Beaudart C, McCloskey E, Bruyère $O$, Cesari M, Rolland $Y$, Rizzoli R, et al. Sarcopenia in daily practice: assessment and management. BMC Geriatr. 2016;16(1):170. https://doi.org/10.1186/s12877-016-0349-4.

16. Goodpaster BH, Kelley DE, Thaete FL, He J, Ross R. Skeletal muscle attenuation determined by computed tomography is associated with skeletal muscle lipid content. J Appl Physiol (1985). 2000;89(1):104-10. https://doi.org/10.1152/jappl.2000.89.1.104

17. Ikemoto-Uezumi M, Matsui $Y$, Hasegawa M, Fujita R, Kanayama $Y$, Uezumi A et al. Disuse atrophy accompanied by intramuscular ectopic ladipogenesis in vastus medialis Musclevastus medialis muscle of advanced osteoarthritis patients. Am J Pathol. 2017;187(12):2674-85. https://doi.org/10.1016/j.ajpa th.2017.08.009.

18. Toba K, Kikuchi R, Iwata A, Kozaki K. "Fall risk index" helps clinicians identify high-risk individuals. Jpn Med Assoc J. 2009;52:237-42.

19. Matsui Y, Fujita R, Harada A, Sakurai T, Nemoto T, Noda N, et al. Association of grip strength and related indices with independence of activities of daily living in older adults, investigated by a newly-developed grip strength measuring device. Geriatr Gerontol Int. 2014;14(Suppl 2):77-86. https://doi. org/10.1111/ggi.12262

20. Fujita R, Matsui Y, Harada A, Takemura M, Kondo I, Nemoto T, et al. Does the $\mathrm{Q}-\mathrm{H}$ index show a stronger relationship than the $\mathrm{H}: \mathrm{Q}$ ratio in regard to knee pain during daily activities in patients with knee osteoarthritis? J Phys Ther Sci. 2016;28:3320-4 doi: 10.1589\%2Fjpts.28.3320.

21. Nakamura K, Ogata T. Locomotive syndrome: Definition and Management Clin Rev Bone Miner Metab. 2016;14:56-67 doi: 10.1007\%2Fs12018-0169208-2.

22. Guralnik JM, Simonsick EM, Ferrucci L, Glynn RJ, Berkman LF, Blazer DG, et al. A short physical performance battery assessing lower extremity function: association with self-reported disability and prediction of mortality and nursing home Admission.nursing home admission. J Gerontol. 1994:49: 85-94. https://doi.org/10.1093/geronj/49.2.M85.

23. Dunn OJ, Clark V. Correlation Coefficients Measured on the Same Individuals. J Am Stat Assoc. 64:325, 366-77. https://doi.org/10.1080/016214 59.1969.10500981

24. Cohen J. Statistical power analysis for the behavioral sciences. 2nd ed. Hillsdale: Lawrence Erlbaum Associates; 1988.

25. McGregor RA, Cameron-Smith D, Poppitt SD. It is not just muscle mass: a review of muscle quality, composition and metabolism during ageing as determinants of muscle function and mobility in later life. Longev Heal. 2014;3:1-8.

26. Heymsfield SB, Gonzalez MC, Lu J, Jia G, Zheng J. Skeletal muscle mass and quality: evolution of modern measurement concepts in the context of sarcopenia. Proc Nutr Soc. 2015;74(4):355-66. https://doi.org/10.1017/S002 9665115000129

27. Chalhoub D, Boudreau R, Greenspan S, Newman AB, Zmuda J, Frank-Wilson $A W$, et al. Associations between lean mass, muscle strength and power, and skeletal size, density and strength in older men. J Bone Miner Res. 2018; 33(9):1612-21. https://doi.org/10.1002/jbmr.3458

28. Visser M, Kritchevsky SB, Goodpaster BH, Newman AB, Nevitt M, Stamm E, et al. Leg muscle mass and composition in relation to lower extremity performance in men and women aged 70 to 79 : the health, aging and body composition study. J Am Geriatr Soc. 2002;50(5):897-904. https://doi. org/10.1046/j.1532-5415.2002.50217.x

29. Lang T, Cauley JA, Tylavsky F, Bauer D, Cummings S, Harris TB, et al. Study. Computed tomographic measurements of thigh muscle cross-sectional area and attenuation coefficient predict hip fracture: the health, aging, and body 
composition study. J Bone Miner Res. 2010;25(3):513-9. https://doi.org/10.13 59/jbmr.090807.

30. Aubrey J, Esfandiari N, Baracos VE, Buteau FA, Frenette J, Putman CT, et al. Measurement of skeletal muscle radiation attenuation and basis of its biological variation. Acta Physiol. 2014;210(3):489-97. https://doi.org/1 0.1111/apha.12224.

31. Marcus RL, Addison O, Dibble LE, Foreman KB, Morrell G, Lastayo P. Intramuscular adipose tissue, sarcopenia, and mobility function in older individuals. J Aging Res. 2012;2012:629637-6. https://doi.org/10.1155/2012/ 629637.

32. Manini TM, Clark BC, Nalls MA, Goodpaster BH, Ploutz-Snyder LL, Harris TB, et al. Reduced physical activity increases intermuscular adipose tissue in healthy young adults. Am J Clin Nutr. 2007;85(2):377-84. https://doi.org/10.1 093/ajcn/85.2.377.

33. Fearon K, Strasser F, Anker SD, Bosaeus I, Bruera E, Fainsinger RL, et al. Definition and classification of cancer cachexia: an international consensus. Lancet Oncol. 2011;12(5):489-95. https://doi.org/10.1016/S1470-2045(1 0)70218-7.

34. Kasai T, Ishiguro N, Matsui Y, Harada A, Takemura M, Yuki A, et al. Sex- and age-related differences in mid-thigh composition and muscle quality determined by computed tomography in middle-aged and elderly Japanese. Geriatr Gerontol Int. 2015;15(6):700-6. https://doi.org/10.1111/ ggi.12338.

35. Schweitzer L, Geisler C, Pourhassan M, Braun W, Glüer CC, Bosy-Westphal A, et al. What is the best reference site for a single MRI slice to assess whole body skeletal muscle and adipose tissue volumes in healthy adults? Am J Clin Nutr. 2015;102(1):58-65. https://doi.org/10.3945/ajcn.115.111203.

36. Lee SJ, Janssen I, Heymsfield SB, Ross R. Relation between whole-body and regional measures of human skeletal muscle. Am J Clin Nutr. 2004;80(5): 1215-21. https://doi.org/10.1093/ajcn/80.5.1215.

37. Tsukasaki K, Matsui Y, Arai H, Harada A, Tomida M, Takemura M, et al. Association of muscle strength and gait speed with cross-sectional muscle area determined by mid-thigh computed tomography - a comparison with skeletal muscle mass measured by dual-energy $\mathrm{x}$-ray absorptiometry. J Frailty Aging. 2020;9(2):2019 doi: https://doi.org/10.14283/ffa.2020.16.

38. Leenders M, Verdijk LB, van der Hoeven L, van Kranenburg J, Nilwik R, van Loon $L$. Elderly men and women benefit equally from prolonged resistance-type exercise training. J Gerontol A Biol Sci Med Sci. 2013;68(7): 769-79. https://doi.org/10.1093/gerona/gls241.

39. Goodpaster BH, Thaete FL, Kelley DE. Composition of skeletal muscle evaluated with computed tomography. Ann N Y Acad Sci. 2000;904(1):1824. https://doi.org/10.1111/j.1749-6632.2000.tb06416.x.

\section{Publisher's Note}

Springer Nature remains neutral with regard to jurisdictional claims in published maps and institutional affiliations.

Ready to submit your research? Choose BMC and benefit from:

- fast, convenient online submission

- thorough peer review by experienced researchers in your field

- rapid publication on acceptance

- support for research data, including large and complex data types

- gold Open Access which fosters wider collaboration and increased citations

- maximum visibility for your research: over $100 \mathrm{M}$ website views per year

At BMC, research is always in progress.

Learn more biomedcentral.com/submissions 